TRANSACTIONS OF THE

AMERICAN MATHEMATICAL SOCIETY

Volume 365, Number 10, October 2013, Pages 5051-5069

S 0002-9947(2013)05675-9

Article electronically published on January 9, 2013

\title{
THETA-DUALITY ON PRYM VARIETIES AND A TORELLI THEOREM
}

\author{
MARTÍ LAHOZ AND JUAN CARLOS NARANJO
}

\begin{abstract}
Let $\pi: \widetilde{C} \rightarrow C$ be an unramified double covering of irreducible smooth curves and let $P$ be the attached Prym variety. We prove the schemetheoretic theta-dual equalities in the Prym variety $T(\widetilde{C})=V^{2}$ and $T\left(V^{2}\right)=\widetilde{C}$, where $V^{2}$ is the Brill-Noether locus of $P$ associated to $\pi$ considered by Welters. As an application we prove a Torelli theorem analogous to the fact that the symmetric product $D^{(g)}$ of a curve $D$ of genus $g$ determines the curve.
\end{abstract}

\section{INTRODUCTION}

Prym varieties are principally polarized abelian varieties (ppav in the sequel) naturally attached to étale double coverings of curves. In analogy with the Jacobian case, there exists two varieties of divisors $X^{+}, X^{-}$playing the role of the symmetric products $C^{(g-1)}, C^{(g)}$ for a Jacobian variety $J C$ of a curve of genus $g$. Indeed both have natural maps to (torsors of) the Prym variety and these maps are the corresponding Albanese maps. The analogy mentioned above comes from the behaviours of these morphisms: while the image of $X^{+}$is a translate of the Prym Theta divisor $\Xi, X^{-}$maps birationally onto the Prym variety. In $[\mathrm{Na}$ and $[\mathrm{SV}]$ it is proved that (under some mild conditions) the variety $X^{+}$determines the covering. One of the goals of this paper is to prove that this is so for $X^{-}$.

This connects with the geometry of the Brill-Noether loci $V^{i}$ of the Prym variety (introduced by Welters [W1, (1.2)]) and its scheme-theoretic theta-dual. To give an idea, $V^{1}$ is a canonical model of Theta divisor $\Xi$, while $V^{3}$ corresponds to the stable singularities of $\Xi$. Instead, $V^{2}$ has not such a natural description in terms of the Theta divisor. It is remarkable that $V^{2}$ is exactly the subscheme of $P$ where the map $X^{-} \rightarrow P$ has positive dimensional fibers. Following Pareschi and Popa (see [PP, Def. 4.2]), we use the Fourier-Mukai transform to provide a natural scheme structure on the theta-dual set $T(X)=\{a \in P \mid a+X \subset \Xi\}$. Actually the definition of $T(Y)$ may not deserve the name (already established) of "thetadual" since the natural inclusion $Y \subset T(T(Y))$ is usually not an equality. The computation of theta-dual sets is a classical subject for Jacobians and Pryms. For example, the equality $T\left(W_{d}\right)=W_{g-d-1}$ is well known and Welters ([W2, W4] ) has studied $T(\operatorname{Sing}(\Theta))$ for Jacobians and for generic Prym varieties (of high enough dimension in the second case). In particular, for a generic Prym variety, he proved

Received by the editors December 1, 2010 and, in revised form, May 26, 2011 and July 25, 2011.

2010 Mathematics Subject Classification. Primary 14Kxx; Secondary 14Hxx.

Both authors have been partially supported by the Proyecto de Investigación MTM2009-14163C02-01. This paper was revised while the first-named author was supported by the SFB/TR 45 'Periods, Moduli Spaces and Arithmetic of Algebraic Varieties' of the DFG (German Research Foundation). 
that the component of dimension 2 of $T\left(V^{3}\right)=T(\operatorname{Sing}(\Xi))$ is the surface $\widetilde{C}-\widetilde{C}$. Debarre proved that for the covering of a generic tetragonal curve of high genus, one has the equality $T\left(V^{3}\right)=T\left(\operatorname{Sing}_{s t}(\Xi)\right)=\widetilde{C}-\widetilde{C}$ (see [D1, Cor. 4.6]).

We prove the following theorems:

Theorem A. Let $\widetilde{C} \rightarrow C$ be an irreducible unramified double covering of a smooth complete non-hyperelliptic irreducible curve of genus $g \geq 4$, and let $(P, \Xi)$ be its Prym variety. Then, the scheme-theoretic equalities $T(\widetilde{C})=V^{2}$ and $T\left(V^{2}\right)=\widetilde{C}$ hold.

For Prym varieties of dimension $4(g=5)$, a set-theoretic version of the previous theorem is proven in [Iz, Thm. 1].

Theorem B. Let $\widetilde{C} \rightarrow C$ be an irreducible unramified double covering of a smooth complete non-hyperelliptic irreducible curve of genus $g>4$, and let $(P, \Xi)$ be its Prym variety. Then the variety $X^{-}(\widetilde{C}, C)$ determines the covering $\widetilde{C} \rightarrow C$.

In fact, we will see that the principal polarization $\Xi$ and $V^{2}$ determine the covering and $X^{-}$determines $V^{2}$ and $\Xi$. We point out that the principal polarization does not determine in general the covering (see [Do, Prop. 1.1] and [IL, Theorem $0.1])$.

Recall that when $C$ is hyperelliptic, the Prym variety is a Jacobian or a product of Jacobians (see [M2, p. 344]) and the statements above are not interesting from the point of view of Prym theory.

A few words about the method of the proofs: fix an unramified double covering $\widetilde{C} \rightarrow C$ (C non-hyperelliptic of genus $g$ ); there is an embedding in the Prym variety $j: \widetilde{C} \rightarrow P$. Analogous with the Jacobian case, one can consider the Fourier-Mukai transform of $j_{*} L$, where $L \in \operatorname{Pic}^{2 g-2}(\widetilde{C})$ has norm $\omega_{C}$. When $h^{0}(\widetilde{C}, L)$ is even, this transform is (quasi-isomorphic to) a sheaf whose projectivization is $X^{+}$(see $\mathrm{Na}$, Thm. 2.1]). Instead, when $h^{0}(L)$ is odd, the Fourier-Mukai transform $\mathbf{R} \mathscr{S}_{P}\left(j_{*} L\right)$ is a complex with two non-vanishing cohomology sheaves $\mathcal{R}^{0}$ and $\mathcal{R}^{1}$. One of the main points of the paper is the isomorphism $\mathcal{R}^{1} \cong \mathcal{I}_{V^{2}}\left(\Xi_{\lambda}\right)$. The scheme-theoretic equality $T\left(V^{2}\right)=\widetilde{C}$ follows quickly (and also the Torelli-like theorem corresponding to $X^{-}$). To prove the isomorphism $\mathcal{R}^{1} \cong \mathcal{I}_{V^{2}}\left(\Xi_{\lambda}\right)$, we combine the use of standard properties of the Fourier-Mukai transform with the opposite equality $T(\widetilde{C})=V^{2}$. Finally, the proof of this last statement (set-theoretically very easy) follows from a delicate argument involving Fitting supports.

It is quite surprising, at least for the authors, that the Brill-Noether locus $V^{2}$ determines the covering without genericity hypothesis or restrictions on the genus. In the last section, this fact allows us to illustrate Theorem $\mathrm{A}$ in a very well-known context: the intermediate Jacobian $J$ of a smooth cubic threefold $V$, since $J$ is isomorphic (as ppav) to a Prym variety of dimension 5 .

\section{Notation AND PRELIMINARIES}

All the varieties are defined over an algebraically closed field of characteristic $\neq$ 2 .

1.1. Prym varieties. We recall some basic facts regarding the theory of Prym varieties. We quote [M2] for the details. 
Let $\pi: \widetilde{C} \rightarrow C$ be an irreducible unramified double covering of an irreducible projective smooth curve $C$ of genus $g$. The kernel of the norm map

$$
N m_{\pi}: J \widetilde{C} \rightarrow J C
$$

has two irreducible components. The component containing the origin is an abelian variety, $P=P(\widetilde{C}, C)$ of dimension $g-1$, called the Prym variety of the covering. We denote by $P^{\prime}$ the other component. The principal polarization on $J \widetilde{C}$ restricts to twice a principal polarization on $P$ and an effective divisor representing the polarization (determined up to translation) is denoted by $\Xi$. Therefore $(P, \Xi)$ is a ppav. Identifying $P$ with its dual using the principal polarization $\Xi$ or dualizing $N m_{\pi}$, we can also consider $P \subset \operatorname{ker}\left(\mathrm{Pic}^{0} \widetilde{C} \rightarrow \mathrm{Pic}^{0} C\right)$.

We will fix a point $\tilde{c} \in \widetilde{C}$ and we will denote by $\sigma$ the involution in $\widetilde{C}$ associated to the covering. We define $j: \widetilde{C} \rightarrow P$ to be the embedding given by $j(\tilde{x})=$ $\mathcal{O}_{\widetilde{C}}(\tilde{x}-\sigma \tilde{x}+\tilde{c}-\sigma \tilde{c})$.

The theta divisor can be defined canonically in

$$
P^{+}=P^{+}(\widetilde{C}, C):=\left\{L \in \operatorname{Pic}^{2 g-2}(\widetilde{C}) \mid N m_{\pi}(L)=\omega_{C}, h^{0}(\widetilde{C}, L) \text { even }\right\},
$$

as $\Xi^{+}=\left\{L \in P^{+} \mid h^{0}(\widetilde{C}, L)>0\right\}$. Throughout this paper, an element $M^{+} \in$ $P^{+}$is fixed and $\Xi$ denotes the divisor in $P$ obtained by translating $\Xi^{+}$by $M^{+}$. In symbols: $\Xi=t_{M^{+}}^{*}\left(\Xi^{+}\right)$, where $t_{M^{+}}: P^{+} \rightarrow P$ stands for the isomorphism $t_{M^{+}}(L)=L \otimes\left(M^{+}\right)^{-1}$ in Pic $\widetilde{C}$.

The Jacobi inversion theorem for Prym varieties (see, e.g. [Na, Lemma 3.2], or [La, III.2.4]) reads:

$$
j^{*}(\Xi)=\sigma^{*}\left(M^{+}\right)(\sigma \tilde{c}-\tilde{c}) .
$$

Let $X^{+}=X^{+}(\widetilde{C}, C), X^{-}=X^{-}(\widetilde{C}, C)$ be the varieties of special divisors defined by

$$
\begin{aligned}
& X^{+}=\left\{D \in \widetilde{C}^{(2 g-2)}\left|\pi^{(2 g-2)}(D) \in\right| \omega_{C} \mid \text { and } h^{0}\left(\widetilde{C}, \mathcal{O}_{\widetilde{C}}(D)\right) \equiv 0 \bmod 2\right\} \text {, } \\
& X^{-}=\left\{D \in \widetilde{C}^{(2 g-2)}\left|\pi^{(2 g-2)}(D) \in\right| \omega_{C} \mid \text { and } h^{0}\left(\widetilde{C}, \mathcal{O}_{\widetilde{C}}(D)\right) \equiv 1 \bmod 2\right\} .
\end{aligned}
$$

Observe that the natural map $\widetilde{C}^{(2 g-2)} \rightarrow \operatorname{Pic}^{2 g-2}(\widetilde{C})$ sends $X^{+}$and $X^{-}$to $P^{+}$ and $P^{-}$respectively. In the even case, $X^{+}$maps onto $\Xi^{+}$. The main result in $\mathrm{Na}$ and $[\mathrm{SV}]$ is that, under some conditions, $X^{+}$determines the covering. Instead $X^{-} \rightarrow P^{-}$is birational.

Definition 1.1. We define the scheme-theoretical Brill-Noether loci in Prym varieties following Welters (see [W1, (1.2)]1]),

$$
\begin{array}{ll}
V^{r}:=W_{g(\widetilde{C})-1}^{r}(\widetilde{C}) \cap P^{+} & \text {if } r \text { is odd, } \\
V^{r}:=W_{g(\widetilde{C})-1}^{r}(\widetilde{C}) \cap P^{-} & \text {if } r \text { is even. }
\end{array}
$$

Observe that as a set, if $r$ is even (resp. odd), then $V^{r} \subset P^{-}\left(\right.$resp. $\left.V^{r} \subset P^{+}\right)$ is

$$
V^{r}=\left\{L \in N m_{\pi}^{-1}\left(\omega_{C}\right) \mid h^{0}(L) \geq r+1, h^{0}(L) \equiv r+1 \quad \bmod 2\right\} .
$$

\footnotetext{
${ }^{1}$ We recall that De Concini and Pragacz $\mathrm{dCP}$. Def. 1] define a more reduced natural scheme structure on $V^{r}$.
} 
For example, the first odd cases are $V^{1}=\Xi^{+} \subset P^{+}=V^{-1}$ and $V^{3}=$ $\operatorname{Sing}_{s t}(\Xi) \subset P^{+}$, the stable singularities of $\Xi$ (see [M2, p. 343]). The first even cases are $V^{0}=P^{-}$and $V^{2}=T(\widetilde{C}) \subset P^{-}$as we will see next.

If $C$ is not hyperelliptic of genus $g \geq 4$, then the codimension of $V^{2}$ in $P^{-}$is 3 (see CLV, Thm. 2.2]). In fact, we will see that if $g>4$, then $V^{2}$ is pure-dimensional, reduced and Cohen-Macaulay (see Lemma 4.1).

1.2. Fourier-Mukai transform. Let $(A, \Theta)$ be a ppav. Consider $A \times A$ with the corresponding projections $p_{1}$ and $p_{2}$ and $m: A \times A \rightarrow A$ the group law. We denote by $\mathcal{M}=m^{*} \mathcal{O}_{A}(\Theta) \otimes p_{1}^{*} \mathcal{O}_{A}(-\Theta) \otimes p_{2}^{*} \mathcal{O}_{A}(-\Theta)$ the Mumford line bundle. Then we define

$$
\mathbf{R} \mathscr{S}_{A}(\mathcal{F}):=\mathbf{R} p_{1 *}\left(p_{2}^{*} \mathcal{F} \otimes \mathcal{M}\right)
$$

the Fourier-Mukai transform $\mathbf{R} \mathscr{S}_{A}: \mathbf{D}^{b}(A) \rightarrow \mathbf{D}^{b}(A)$, where $\mathbf{D}^{b}(A)$ is the bounded derived category of coherent sheaves on $A$. Due to the well-known theorem of Mukai [Mu1, Thm. 2.2], this is an equivalence of categories.

By base-change one has an inclusion of sets,

$$
\operatorname{supp} R^{i} \mathscr{S}_{A}(\mathcal{F}) \subset V^{i}(\mathcal{F}):=\left\{\alpha \in \widehat{A} \mid h^{i}(\mathcal{F} \otimes \alpha)>0\right\} .
$$

The closed sets $V^{i}(\mathcal{F})$ are called the cohomological support loci attached to $\mathcal{F}$.

A sheaf $\mathcal{F}$ on $X$ satisfies the Weak Index Theorem $\left(\operatorname{WIT}\left(i_{0}\right)\right)$ if there exists $0 \leq i_{0} \leq \operatorname{dim} X$ such that $R^{i} \mathscr{S}_{A}(\mathcal{F})=0, \forall i \neq i_{0}$ and $R^{i_{0}} \mathscr{S}_{A}(\mathcal{F}) \neq 0$.

We will use the usual dualizing functor in the derived category of a smooth scheme $X$ over a field,

$$
\mathbf{R} \Delta_{X} \mathcal{F}=\mathbf{R} \mathcal{H o m}\left(\mathcal{F}, \omega_{X}\right)
$$

Grothendieck-Verdier duality applied to our context (see [Mu1, (3.8)]) says

$$
\mathbf{R} \Delta_{P} \circ \mathbf{R} \mathscr{S}_{P} \cong[g-1] \circ(-1)^{*} \mathbf{R} \mathscr{S}_{P} \circ \mathbf{R} \Delta_{P} .
$$

Also again by Grothendieck-Verdier duality (e.g. [Hu, Thm. 3.34]) we have

$$
\mathbf{R} \Delta_{P} \circ j_{*} \cong[2-g] \circ j_{*} \circ \mathbf{R} \Delta_{\widetilde{C}} .
$$

1.3. Theta-duality. Let $(A, \Theta)$ be a principally polarized abelian variety. Given a morphism $f: T \rightarrow A$, we define the translation $t_{f}$ along $f$ to be the composite

$$
t_{f}: A \times T \stackrel{1 \times f}{\longrightarrow} A \times A \stackrel{m}{\longrightarrow} A,
$$

where $m$ is the group law in $A$.

Definition 1.2. The theta-dual of a closed subscheme $Y \subseteq A$ is the unique closed subscheme $T(Y) \subseteq A$ with the universal property that an arbitrary morphism $f: T \rightarrow A$ factors through $T(Y)$ if and only if $Y \times T \subseteq t_{f}^{-1}(\Theta)$ lies in $A \times T$.

Thus, as a set, $T(Y)$ consists of those points $a \in A$ for which the theta-translate $t_{a}^{-1} \Theta$ contains $Y$ as a scheme. As proved in GL, Prop. 2.5], the theta-dual always exists as a scheme and, by GL, Prop. 2.6], equals the object of the same name defined in Pareschi-Popa [PP, Def. 4.2]. That is, the theta-dual can be computed as the following scheme-theoretic support

$$
T(Y)=\operatorname{supp}\left(-1_{A}\right)^{*} R^{a} \mathscr{S}_{A}\left(\mathbf{R} \Delta_{A}\left(\mathcal{I}_{Y}(\Theta)\right)\right),
$$

where $a=\operatorname{dim} A$. 
We also recall $\left[\mathrm{PP}\right.$, Cor. 4.3]: the sheaf $\left(-1_{A}\right)^{*} R^{a} \mathscr{S}\left(\mathbf{R} \Delta_{A}\left(\mathcal{I}_{Y}(\Theta)\right)\right)$ is a line bundle on $T(Y)$ and

$$
\mathcal{O}_{T(Y)}(\Theta) \cong\left(-1_{A}\right)^{*} R^{a} \mathscr{S}\left(\mathbf{R} \Delta_{A}\left(\mathcal{I}_{Y}(\Theta)\right)\right) \cong \mathcal{H o m}\left(\mathbf{R} \mathscr{S}_{A}\left(\mathcal{I}_{Y}(\Theta)\right), \mathcal{O}_{A}\right) .
$$

The second isomorphism is a consequence of Grothendieck-Verdier duality (see La, Rem. I.1.7] for the details).

1.4. Relation between $\mathbf{R} \mathscr{S}_{J \widetilde{C}}\left(i_{*}(-)\right)$ and $\mathbf{R} \mathscr{S}_{P}\left(j_{*}(-)\right)$. Given a smooth irreducible projective curve, we denote by $i$ an Abel-Jacobi immersion of the curve in its Jacobian attached to a fixed point.

In [La, §III.2.4], Lahoz proves the following generalization of [Na, Prop. 3.1],

$$
\mathbf{R} \mathscr{S}_{P}\left(j_{*}(-)\right) \cong \mathbf{R} \mathscr{S}_{J \widetilde{C}}\left(i_{*}(-)\right) \underline{\otimes} \mathcal{O}_{P} .
$$

A similar result is true with respect to $P^{\prime}$.

Lemma 1.3. We have the following isomorphisms as functors of the derived category of $P^{\prime}$

$$
\mathbf{R} \mathscr{S}_{J \widetilde{C}}\left(i_{*}(-)\right) \underline{\otimes}_{P^{\prime}} \cong t_{-L^{\prime}}^{*}\left(\mathbf{R} \mathscr{S}_{P}\left(j_{*}\left(-\otimes L^{\prime}\right)\right)\right),
$$

where $L^{\prime}=\mathcal{O}_{\widetilde{C}}(\sigma \tilde{c}-\tilde{c})$.

Proof. Observe that $\mathbf{R} \mathscr{S}_{J \widetilde{C}}\left(i_{*}(-)\right) \underline{\otimes} \mathcal{O}_{P^{\prime}} \cong t_{-L^{\prime}}^{*}\left(t_{L^{\prime}}^{*}\left(\mathbf{R} \mathscr{S}_{J \widetilde{C}}\left(i_{*}(-)\right)\right) \underline{\otimes} \mathcal{O}_{P}\right)$, where $L^{\prime} \in P^{\prime}$ is $L^{\prime} \cong \mathcal{O}_{\widetilde{C}}(\sigma \tilde{c}-\tilde{c})$. Then

$$
\begin{aligned}
\mathbf{R} \mathscr{S}_{J \widetilde{C}}\left(i_{*}(-)\right) \underline{\otimes} \mathcal{O}_{P^{\prime}} \cong t_{-L^{\prime}}^{*} & \left.\left(\mathbf{R} \mathscr{S}_{J \widetilde{C}}\left(i_{*}(-) \otimes L^{\prime}\right)\right) \otimes \mathcal{O}_{P}\right) \\
& \left.\cong t_{-L^{\prime}}^{*}\left(\mathbf{R} \mathscr{S}_{J \widetilde{C}}\left(i_{*}\left(-\otimes L^{\prime}\right)\right)\right) \underline{\otimes}_{P}\right) \\
& \cong t_{-L^{\prime}}^{*}\left(\mathbf{R} \mathscr{S}_{P}\left(j_{*}\left(-\otimes L^{\prime}\right)\right)\right) .
\end{aligned}
$$

We consider an invertible sheaf on $\widetilde{C}$ with norm the canonical sheaf and odd $h^{0}$, i.e. $L^{-} \in P^{-}$. The sheaf $j_{*} L^{-}$on $P$ is supported on the curve $\widetilde{C}$ and its FourierMukai transform codifies geometric information of the ppav $(P, \Xi)$ in connection with the subvariety $V^{2}$. The first observation is that this sheaf is not WIT (compare with the situation when one takes $L^{+} \in P^{+}$as studied in [Na, 3.4]).

Observe that the cohomological support loci attached to $j_{*} L^{-}$are

$$
V^{0}\left(j_{*} L^{-}\right)=V^{1}\left(j_{*} L^{-}\right)=P, \quad V^{i}\left(j_{*} L^{-}\right)=\emptyset \text { for } i \geq 2 .
$$

Moreover $R^{0} \mathscr{S}_{P}\left(j_{*} L^{-}\right), R^{1} \mathscr{S}_{P}\left(j_{*} L^{-}\right)$have (generic) rank 1 and $R^{i} \mathscr{S}_{P}\left(j_{*} L^{-}\right)=0$ for $i \geq 2$.

Observe that $R^{1} \mathscr{S}_{P}\left(j_{*}\left(L^{-}\right)\right.$is the highest non-vanishing sheaf, so it has the "base change property"; in other words,

$$
R^{1} \mathscr{S}_{P}\left(j_{*} L^{-}\right) \otimes k(\alpha) \cong H^{1}\left(\widetilde{C}, L^{-} \otimes \alpha\right) \cong H^{0}\left(\widetilde{C}, \sigma^{*} L^{-} \otimes \alpha^{-1}\right)^{*} .
$$

Hence it is an invertible sheaf on the complement of (some translate) of $V^{2}$ in $P$.

Corollary 1.4. We have the following sheaf isomorphisms:

$$
R^{1} \mathscr{S}_{J \widetilde{C}}\left(i_{*} L^{-}\right)_{\mid P} \cong R^{1} \mathscr{S}_{P}\left(j_{*} L^{-}\right),
$$

and

$$
\begin{gathered}
\mathcal{T}_{\operatorname{or}_{1}}\left(R^{1} \mathscr{S}_{J \widetilde{C}}\left(i_{*} L^{-}\right), \mathcal{O}_{P}\right) \cong R^{0} \mathscr{S}_{P}\left(j_{*} L^{-}\right), \\
R^{1} \mathscr{S}_{J \widetilde{C}}\left(i_{*} L^{-}\right)_{\mid P^{\prime}} \cong t_{-L^{\prime}}^{*}\left(R^{1} \mathscr{S}_{P}\left(j_{*}\left(L^{-} \otimes L^{\prime}\right)\right)\right)
\end{gathered}
$$


for any $L^{-} \in P^{-} \subset \operatorname{Pic}^{2 g-2} \widetilde{C}$. Moreover

$$
\mathcal{I}_{V^{2} / P}=\operatorname{Fitt}_{2}\left(R^{1} \mathscr{S}_{P}\left(j_{*} M^{+}\right)\right),
$$

where $M^{+}$is the element used to translate $\Xi^{+}$into $P$.

Proof. Since an element $L \in \operatorname{Pic}^{2 g-2} \widetilde{C}$ satisfies WIT(1) with respect to $\mathbf{R} \mathscr{S}_{J \widetilde{C}}$ and an element of $L^{+}$satisfies WIT(1) with respect to $\mathbf{R} \mathscr{S}_{P}$, the equalities follow directly from the previous lemma. The last statement follows by definition.

\section{Scheme theoretical Theta-dual of the Abel-Prym Curve}

The aim of this section is to prove the first part of Theorem A namely the scheme theoretical equality $T(\widetilde{C})=V^{2}$. We assume $g \geq 4$.

Lemma 2.1. We have the set-theoretic equality

$$
T(\widetilde{C})=V^{2} .
$$

Proof. An element $L \in P^{-}$belongs to $T(\widetilde{C})$ if and only if $\widetilde{C} \subset \Xi_{-L}^{+}$, which, by the definition of $\widetilde{C} \subset P^{\prime}$, is equivalent to $h^{0}\left(\widetilde{C}, L \otimes \mathcal{O}_{\widetilde{C}}(\sigma p-p)\right)>0$ for every $p \in \widetilde{C}$. By Mumford's parity trick, this happens if and only if $h^{0}(\widetilde{C}, L) \geq 3$; that is, $L \in V^{2}$. Indeed, for a generic $p \in \widetilde{C}, h^{0}(\widetilde{C}, L)=h^{0}\left(\widetilde{C}, L \otimes \mathcal{O}_{\widetilde{C}}(\sigma p-p)\right)+1$ (see [M1, p. 188, Step II]). Since $L \in P^{-}$, then $h^{0}\left(\widetilde{C}, L \otimes \mathcal{O}_{\widetilde{C}}(\sigma p-p)\right)>0$ for every $p \in \widetilde{C}$ if and only if $L \in V^{2}$.

The remainder of this section is devoted to proving the following result.

Theorem 2.2. We have the scheme-theoretic equality

$$
T(\widetilde{C})=V^{2} .
$$

By Lemma 2.1 we already know that the set theoretical equality holds in $P^{-}$. Now, in order to avoid all the translations that could complicate the Fourier-Mukai argument, we will work up to the translation on $P$ using the fixed isomorphisms.

We know by (2),

$$
\mathcal{O}_{T(\widetilde{C})}(\Xi)=\mathcal{H o m}\left(\mathbf{R} \mathscr{S}_{P}\left(\mathcal{I}_{\widetilde{C}}(\Xi)\right), \mathcal{O}_{P}\right),
$$

so we want to compute this last sheaf, or construct a comprehensible short exact sequence where it appears.

Consider $\tilde{c}$ the point in $\widetilde{C}$ that we have used to define the Abel-Prym map $j$ and the following short exact sequence in $P$,

$$
0 \rightarrow \mathcal{I}_{\widetilde{C} / P}(\Xi) \rightarrow \mathcal{I}_{0 / P}(\Xi) \rightarrow j_{*} \mathcal{I}_{\sigma(\tilde{c}) / \widetilde{C}}(\Xi) \rightarrow 0
$$

where we have that $j(\sigma(\tilde{c}))=0 \in P$. We will work out the Fourier-Mukai transform of this exact sequence and its dual. The transforms of the leftmost sheaf will provide the sheaves that we want to understand. The middle sheaf is easy to work with. And the rightmost sheaf can be worked out in the Abel-Prym curve. We get the following lemma.

Lemma 2.3. The sheaves $\mathcal{I}_{0 / P}(\Xi)$ and $j_{*} \mathcal{I}_{\sigma(\tilde{c}) / \widetilde{C}}(\Xi)$ satisfy WIT(1). More precisely

$\mathbf{R} \mathscr{S}_{P}\left(\mathcal{I}_{0 / P}(\Xi)\right)=\mathcal{O}_{\Xi}[-1], \quad \mathbf{R} \mathscr{S}_{P} j_{*} \mathcal{I}_{\sigma(\tilde{c}) / \widetilde{C}}(\Xi)=R^{1} \mathscr{S}_{P}\left(j_{*}\left(\sigma^{*}\left(M^{+}\right)(-\tilde{c})\right)\right)[-1]$, where $M^{+} \in P^{+}$satisfies $\Xi=t_{M^{+}}^{*}\left(\Xi^{+}\right)$. 
Proof. The statement on $\mathcal{I}_{0 / P}(\Xi)$ comes easily since

$$
\mathbf{R} \mathscr{S}_{P}\left(\mathcal{O}_{P}(\Xi)\right)=\mathcal{O}_{P}(-\Xi)[0], \quad \mathbf{R} \mathscr{S}_{P}(k(0))=\mathcal{O}_{P}[0]
$$

and $R^{0} \mathscr{S}_{P}\left(\mathcal{I}_{0 / P}(\Xi)\right)$ is a torsion sheaf.

On the other hand, by (1), we have

$$
j_{*} \mathcal{I}_{\sigma(\tilde{c}) / \widetilde{C}}(\Xi)=j_{*} \mathcal{I}_{\sigma(\tilde{c}) / \widetilde{C}}\left(j^{*}(\Xi)\right)=j_{*}\left(\sigma^{*}\left(M^{+}\right)(-\tilde{c})\right) .
$$

Therefore, by applying $\mathbf{R} \mathscr{S}_{P} \circ j_{*}$ to the short exact sequence,

$$
0 \rightarrow \sigma^{*}\left(M^{+}\right)(-\tilde{c}) \rightarrow \sigma^{*}\left(M^{+}\right) \rightarrow k(\tilde{c}) \rightarrow 0,
$$

and using that $j_{*} \sigma^{*} M^{+}$satisfies $\operatorname{WIT}(1)$ (see [Na, 3.4]) we are done.

We apply the Fourier-Mukai transform to (4) and we implement the information obtained in the lemma above. We get that $R^{0} \mathscr{S}_{P}\left(\mathcal{I}_{\widetilde{C} / P}(\Xi)\right)=0$ and the following exact sequence

$$
0 \rightarrow R^{1} \mathscr{S}_{P}\left(\mathcal{I}_{\widetilde{C} / P}(\Xi)\right) \rightarrow \mathcal{O}_{\Xi} \rightarrow R^{1} \mathscr{S}_{P}\left(j_{*}\left(\sigma^{*}\left(M^{+}\right)(-\tilde{c})\right)\right) \rightarrow R^{2} \mathscr{S}_{P}\left(\mathcal{I}_{\widetilde{C} / P}(\Xi)\right) \rightarrow 0
$$

By [CLV, Thm. 2.2 and Thm. 3.1], the first sheaf of this sequence is supported in codimension at least 3 , hence it vanishes. We get the following.

Corollary 2.4. The sheaf $\mathcal{I}_{\widetilde{C} / P}(\Xi)$ satisfies $W I T(2)$ and there is a short exact sequence

$$
0 \rightarrow \mathcal{O}_{\Xi} \rightarrow R^{1} \mathscr{S}_{P}\left(j_{*}\left(\sigma^{*}\left(M^{+}\right)(-\tilde{c})\right)\right) \rightarrow R^{2} \mathscr{S}_{P}\left(\mathcal{I}_{\widetilde{C} / P}(\Xi)\right) \rightarrow 0
$$

An interesting consequence of this corollary is as follows.

Corollary 2.5. The following isomorphism holds:

$$
\mathcal{E}_{x t^{2}}\left(R^{2} \mathscr{S}_{P}\left(\mathcal{I}_{\widetilde{C} / P}(\Xi)\right), \mathcal{O}_{P}\right)=\mathcal{O}_{T(\widetilde{C})}(\Xi) .
$$

Proof. We apply (2) and the corollary above:

$$
\begin{aligned}
\mathcal{O}_{T(\widetilde{C})}(\Xi) & \cong \mathcal{H o m}\left(\mathbf{R} \mathscr{S}_{P}\left(\mathcal{I}_{\widetilde{C} / P}(\Xi)\right), \mathcal{O}_{P}\right) \\
& \cong \mathcal{H o m}\left(R^{2} \mathscr{S}_{P}\left(\mathcal{I}_{\widetilde{C} / P}(\Xi)\right)[-2], \mathcal{O}_{P}\right) \\
& \cong \mathcal{E} x t^{2}\left(R^{2} \mathscr{S}_{P}\left(\mathcal{I}_{\widetilde{C} / P}(\Xi)\right), \mathcal{O}_{P}\right) .
\end{aligned}
$$

In particular, by [CLV] Thm. 2.2], we have

$$
\operatorname{codim}_{P} \mathcal{E} x t^{2}\left(R^{2} \mathscr{S}_{P}\left(\mathcal{I}_{\widetilde{C} / P}(\Xi)\right), \mathcal{O}_{P}\right) \geq 3 .
$$

Now, we apply the functor $\mathbf{R H o m}\left(\cdot, \mathcal{O}_{P}\right)$ to (5). We get

$$
\mathcal{H o m}\left(R^{2} \mathscr{S}_{P}\left(\mathcal{I}_{\widetilde{C} / P}(\Xi)\right), \mathcal{O}_{P}\right) \cong \mathcal{H o m}\left(R^{1} \mathscr{S}_{P}\left(j_{*}\left(\sigma^{*}\left(M^{+}\right)(-\tilde{c})\right)\right), \mathcal{O}_{P}\right)
$$

and the exact sequence

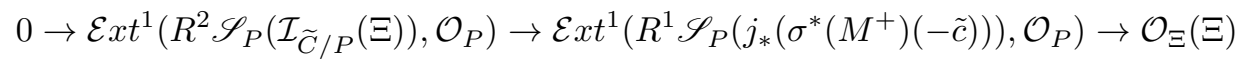

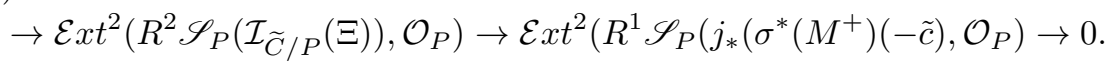

The next step is to prove the vanishing of two of the terms of this exact sequence. This is the content of the next two lemmas.

Lemma 2.6. We have

$$
\mathcal{E x t}^{1}\left(R^{2} \mathscr{S}_{P}\left(\mathcal{I}_{\widetilde{C} / P}(\Xi)\right), \mathcal{O}_{P}\right)=0
$$


Proof. Observe that

$$
\begin{aligned}
\mathcal{E} x t^{1}\left(R^{2} \mathscr{S}_{P}\left(\mathcal{I}_{\widetilde{C} / P}(\Xi)\right), \mathcal{O}_{P}\right) & =\mathcal{E} x t^{-1}\left(\mathbf{R} \mathscr{S}_{P}\left(\mathcal{I}_{\widetilde{C} / P}(\Xi)\right), \mathcal{O}_{P}\right) \\
& =R^{g-2} \mathscr{S}_{P} \mathbf{R} \Delta_{P}\left(\mathcal{I}_{\widetilde{C} / P}(\Xi)\right) .
\end{aligned}
$$

By base change, as a set, $\operatorname{supp} R^{g-2} \mathscr{S}_{P} \mathbf{R} \Delta_{P}\left(\mathcal{I}_{\widetilde{C} / P}(\Xi)\right) \subseteq V^{g-2}\left(\mathbf{R} \Delta\left(\mathcal{I}_{\widetilde{C} / P}(\Xi)\right)\right)=$ $-V^{1}\left(\mathcal{I}_{\widetilde{C} / P}(\Xi)\right)$ (the last equality, follows from Grothendieck-Serre duality), so we have

$$
\operatorname{codim}_{P} \mathcal{E} x t^{1}\left(R^{2} \mathscr{S}_{P}\left(\mathcal{I}_{\widetilde{C} / P}(\Xi)\right), \mathcal{O}_{P}\right) \geq 3
$$

by CLV, Thm. 3.1 and Thm. 2.2].

Since $P$ is smooth, the functor $\mathbf{R H o m}\left(\cdot, \mathcal{O}_{P}\right)$ is an involution on $\mathbf{D}^{b}(P)$. Thus there is a fourth quadrant spectral sequence

$$
\begin{aligned}
& E_{2}^{i, j}:=\mathcal{E} x t^{i}\left(\left(\mathcal{E} x t^{-j}\left(R^{2} \mathscr{S}_{P}\left(\mathcal{I}_{\widetilde{C} / P}(\Xi)\right), \mathcal{O}_{P}\right), \mathcal{O}_{P}\right)\right. \\
& \Rightarrow H^{i+j}= \begin{cases}R^{2} \mathscr{S}_{P}\left(\mathcal{I}_{\widetilde{C} / P}(\Xi)\right) & \text { if } i+j=0, \\
0 & \text { otherwise. }\end{cases}
\end{aligned}
$$

We have the following:

$$
\begin{aligned}
\operatorname{codim}_{P} \operatorname{supp} \mathcal{E} x t^{1}\left(R^{2} \mathscr{S}_{P}\left(\mathcal{I}_{\widetilde{C} / P}(\Xi)\right), \mathcal{O}_{P}\right) & \geq 3, & & \text { by (10), } \\
\operatorname{codim}_{P} \operatorname{supp} \mathcal{E} x t^{2}\left(R^{2} \mathscr{S}_{P}\left(\mathcal{I}_{\widetilde{C} / P}(\Xi)\right), \mathcal{O}_{P}\right) & \geq 3, & & \text { by (7), } \\
\mathcal{E} x t^{i}\left(R^{2} \mathscr{S}_{P}\left(\mathcal{I}_{\widetilde{C} / P}(\Xi)\right), \mathcal{O}_{P}\right) & =0 & \text { for all } i>2 . &
\end{aligned}
$$

Recall that $\mathcal{E} x t^{l}\left(\mathcal{F}, \mathcal{O}_{P}\right)=0$ for all $l<\operatorname{codim}_{P} \operatorname{supp} \mathcal{F}$. Then the previous spectral sequence yields the following exact sequence

$$
\begin{aligned}
0 \rightarrow R^{2} \mathscr{S}_{P}\left(\mathcal{I}_{\widetilde{C} / P}(\Xi)\right) \rightarrow \mathcal{H o m}\left(\mathcal{H o m}\left(R^{2} \mathscr{S}_{P}\left(\mathcal{I}_{\widetilde{C} / P}(\Xi)\right), \mathcal{O}_{P}\right), \mathcal{O}_{P}\right) \\
\rightarrow \mathcal{E x t}^{3}\left(\mathcal{O}_{T(\widetilde{C})}(\Xi), \mathcal{O}_{P}\right) \rightarrow 0
\end{aligned}
$$

It is not hard to see that $R^{1} \mathscr{S}_{P}\left(j_{*}\left(\sigma^{*}\left(M^{+}\right)(-\tilde{c})\right)\right)$ has (generic) rank 1. By (8),

$$
\mathcal{H o m}\left(\mathcal{H o m}\left(R^{2} \mathscr{S}_{P}\left(\mathcal{I}_{\widetilde{C} / P}(\Xi)\right), \mathcal{O}_{P}\right), \mathcal{O}_{P}\right)
$$

is a reflexive sheaf of rank 1 , hence a line bundle. Thus, dualizing (11) we get

$$
\mathcal{E} x t^{1}\left(R^{2} \mathscr{S}_{P}\left(\mathcal{I}_{\widetilde{C} / P}(\Xi)\right), \mathcal{O}_{P}\right)=0 .
$$

Lemma 2.7. We have the vanishing

$$
\mathcal{E} x t^{2}\left(R^{1} \mathscr{S}_{P}\left(j_{*}\left(\sigma^{*}\left(M^{+}\right)(-\tilde{c})\right)\right), \mathcal{O}_{P}\right)=0
$$

and the isomorphism

$$
\mathcal{E} x t^{1}\left(R^{1} \mathscr{S}_{P}\left(j_{*}\left(\sigma^{*}\left(M^{+}\right)(-\tilde{c})\right)\right), \mathcal{O}_{P}\right) \cong(-1)^{*} R^{1} \mathscr{S}_{P}\left(j_{*} M^{+}(\tilde{c})\right) .
$$

In particular, by the previous lemma, (6) and (9), $(-1)^{*} R^{1} \mathscr{S}_{P}\left(j_{*} M^{+}(\tilde{c})\right) \cong$ $\mathcal{I}_{T(\widetilde{C}) / \Xi}(\Xi)$.

Proof. Since $j_{*} \sigma^{*}\left(M^{+}\right)$satisfies $\operatorname{WIT}(1)$ and $k(j(\tilde{c}))$ satisfies $\operatorname{WIT}(0)$, we get from the exact sequence,

$$
0 \rightarrow j_{*}\left(\sigma^{*}\left(M^{+}\right)(-\tilde{c})\right) \rightarrow j_{*} \sigma^{*} M^{+} \rightarrow k(j(\tilde{c})) \rightarrow 0
$$


that $j_{*}\left(\sigma^{*}\left(M^{+}\right)(-\tilde{c})\right)$ satisfies $\operatorname{WIT}(1)$. Therefore

$$
\mathcal{E} x t^{i}\left(R^{1} \mathscr{S}_{P}\left(j_{*}\left(\sigma^{*}\left(M^{+}\right)(-\tilde{c})\right)\right), \mathcal{O}_{P}\right) \cong \mathcal{E} x t^{i}\left(\mathbf{R} \mathscr{S}_{P}\left(j_{*}\left(\sigma^{*}\left(M^{+}\right)(-\tilde{c})\right)\right)[1], \mathcal{O}_{P}\right) .
$$

By Grothendieck-Verdier duality we have

$$
\begin{aligned}
\mathbf{R} \Delta_{P}\left(\mathbf{R} \mathscr{S}_{P}\left(j_{*}\left(\sigma^{*}\left(M^{+}\right)(-\tilde{c})\right)\right)[1]\right) & \cong \mathbf{R} \Delta_{P}\left(\mathbf{R} \mathscr{S}_{P}\left(j_{*}\left(\sigma^{*}\left(M^{+}\right)(-\tilde{c})\right)\right)[-1]\right. \\
& \cong(-1)^{*} \mathbf{R} \mathscr{S}_{P} \mathbf{R} \Delta_{P}\left(j_{*}\left(\sigma^{*}\left(M^{+}\right)(-\tilde{c})\right)\right)[g-2] \\
& \cong(-1)^{*} \mathbf{R} \mathscr{S}_{P} j_{*} \mathbf{R} \Delta_{\widetilde{C}}\left(\sigma^{*}\left(M^{+}\right)(-\tilde{c})\right) \\
& \cong(-1)^{*} \mathbf{R} \mathscr{S}_{P} j_{*} \sigma^{*}\left(M^{+}(\tilde{c})\right) .
\end{aligned}
$$

This implies the statements of the lemma.

End of the proof of Theorem 2.2. We focus on the computation of $R^{1} \mathscr{S}_{P}\left(j_{*} M^{+}(\tilde{c})\right)$. Consider $E$ an effective divisor on $\widetilde{C}$ of sufficiently high degree $m \gg 0$ whose support does not contain $\tilde{c}$ and the following diagram,

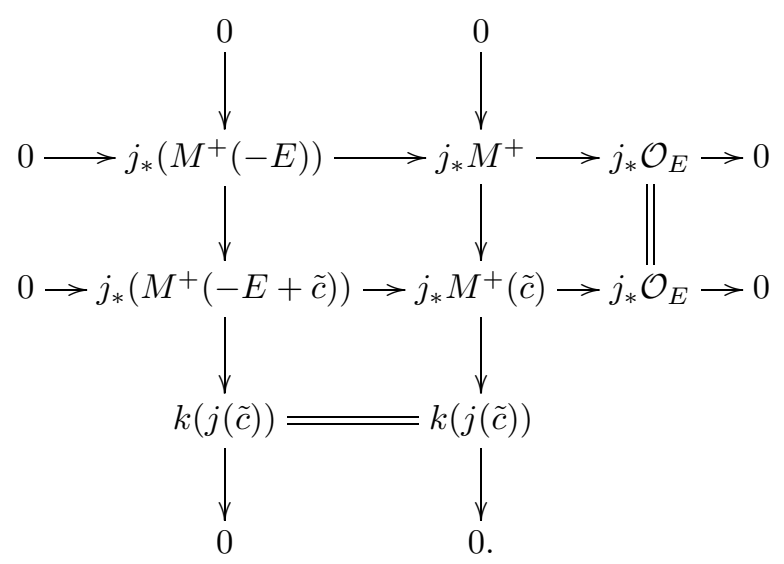

Observe that $j_{*}\left(M^{+}-E\right)$ and $j_{*}\left(M^{+}(\tilde{c})-E\right)$ satisfy the index theorem with index 1 .

Hence, if we apply the Fourier-Mukai transform to the previous diagram we obtain the following commutative diagram,

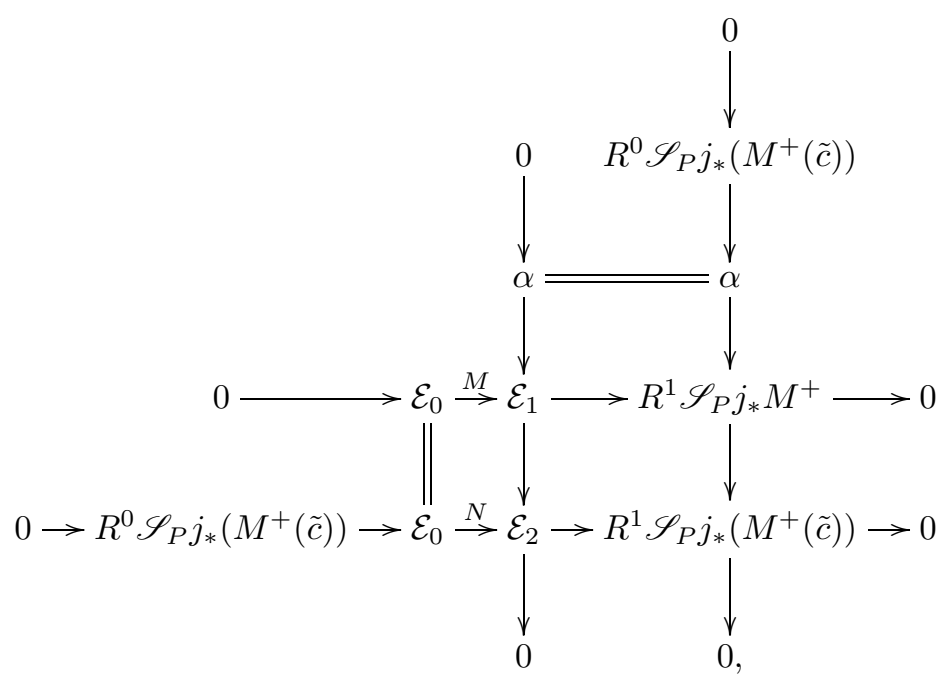


where the sheaves $\mathcal{E}_{i}$ are locally free of rank rk $\mathcal{E}_{0}=\operatorname{rk} \mathcal{E}_{1}=m$ and $\operatorname{rk} \mathcal{E}_{2}=m-1$ and $\alpha \in \operatorname{Pic}^{0} P$.

Recall that $\mathcal{I}_{T(\widetilde{C}) / \Xi}(\Xi)=R^{1} \mathscr{S}_{P}\left(j_{*} M^{+}(\tilde{c})\right)$. Observe that the Fitting ideals can be computed locally since they commute with arbitrary base change. Choose a covering of $P$ by open affine subsets such that, in any of these open subsets, the first vertical short exact sequence of diagram (12) splits. Consider an ordered basis of $\mathcal{E}_{1}$, such that the last vector generates $\alpha$. Then, choose a basis of $\mathcal{E}_{0}$ such that in these open sets the matrices representing $M$ and $N$ are (i.e. choose the counterimage of the generator of $\alpha$ as the last vector in the basis of $\mathcal{E}_{0}$ )

$$
M=\left(\frac{N}{0 \cdots 0 f}\right) .
$$

Hence, $M$ sends the last vector $v=(0, \ldots, 0,1)$ in the basis of $\mathcal{E}_{0}$ to an element of $\alpha$. So, $N$ maps $v$ to 0 and on this basis

$$
N=\left(\begin{array}{ccc|c}
* & \cdots & * & 0 \\
\vdots & A & \vdots & \vdots \\
* & \cdots & * & 0
\end{array}\right)
$$

Then $\mathcal{I}_{T(\widetilde{C}) / P}$ is defined locally by the locus where $N$ drops rank, i.e. the $(m-$ $2) \times(m-2)$ minors of $N$, that coincide with the $(m-2) \times(m-2)$ minors of $A$ and that, multiplied by $f$, are the same of those of $M$. So, locally,

$$
\mathcal{I}_{T(\widetilde{C}) / P} \cdot(f)=\operatorname{Fitt}_{2}\left(R^{1} \mathscr{S}_{P}\left(j_{*} M^{+}\right)\right)=\mathcal{I}_{V^{2} / P} .
$$

Moreover, $\mathcal{I}_{\Xi / P}=\mathcal{I}_{V^{1} / P}=\operatorname{Fitt}_{1}\left(R^{1} \mathscr{S}_{P} j_{*} M^{+}\right)$which is locally defined by $(\operatorname{det} M)$. On the other hand, $\mathcal{I}_{\Xi / P}=\mathcal{I}_{\text {supp } R^{1} \mathscr{S}_{P} j_{*}\left(M^{+}(\tilde{c})\right)}$ which is locally defined by $(\operatorname{det} A)$. Hence $f=1$ and $V^{2}=T(\widetilde{C})$ scheme-theoretically.

\section{Odd Prym Picard sheaves and Theta-dual of $V^{2}$}

In this section we complete the proof of Theorem $\mathrm{A}$. Theorem $\mathrm{B}$ will be an easy consequence.

3.1. Resolutions. From now on, to simplify notation, we put $\mathcal{R}^{0}:=R^{0} \mathscr{S}_{P}\left(j_{*} L^{-}\right)$ and $\mathcal{R}^{1}:=R^{1} \mathscr{S}_{P}\left(j_{*} L^{-}\right)$. Let $E$ be a reduced divisor on $\widetilde{C}$ of degree $m \gg 0$. By applying the functors $j_{*}$ and $\mathbf{R} \mathscr{S}_{P}$ to the exact sequence

$$
0 \rightarrow L^{-} \rightarrow L^{-}(E) \rightarrow L^{-}(E)_{\mid E} \rightarrow 0
$$

we get

$$
\begin{aligned}
0 & \rightarrow \mathcal{R}^{0} \rightarrow R^{0} \mathscr{S}_{P}\left(j_{*}\left(L^{-}(E)\right)\right) \rightarrow R^{0} \mathscr{S}_{P}\left(j_{*}\left(L^{-}(E)_{\mid E}\right)\right) \\
& \rightarrow \mathcal{R}^{1} \rightarrow R^{1} \mathscr{S}_{P}\left(j_{*}\left(L^{-}(E)\right)\right) \rightarrow \ldots
\end{aligned}
$$

One easily checks that $R^{i} \mathscr{S}_{P}\left(j_{*}\left(L^{-}(E)\right)\right)=R^{i} \mathscr{S}_{P}\left(j_{*}\left(L^{-}(E)_{\mid E}\right)\right)=0$ for $i \geq 1$ and that

$$
\begin{aligned}
& \mathcal{E}^{0}:=R^{0} \mathscr{S}_{P}\left(j_{*}\left(L^{-}(E)\right)\right), \\
& \mathcal{G}^{0}:=R^{0} \mathscr{S}_{P}\left(j_{*}\left(L^{-}(E)_{\mid E}\right)\right),
\end{aligned}
$$

are locally free sheaves of rank $m$. In other words, $j_{*}\left(L^{-}(E)\right)$ and $j_{*}\left(L^{-}(E)_{\mid E}\right)$ satisfy $\operatorname{WIT}(0)$ and $\mathcal{E}^{0}, \mathcal{G}^{0}$ are their Fourier-Mukai transforms.

The exact sequence (13) becomes

$$
0 \rightarrow \mathcal{R}^{0} \rightarrow \mathcal{E}^{0} \rightarrow \mathcal{G}^{0} \rightarrow \mathcal{R}^{1} \rightarrow 0 .
$$


For future use we note that $\mathcal{G}^{0}$ is particularly simple since it is the transformation of a sheaf supported on $E$ (since $E$ is reduced, its support is a collection of distinct points $j\left(x_{k}\right), k=1, \ldots, m$ in $P$ ) then,

$$
\mathcal{G}^{0}=\bigoplus_{k=1}^{m} \mathcal{L}_{j\left(x_{k}\right)},
$$

where $\mathcal{L}_{j\left(x_{k}\right)}:=\mathcal{O}_{P}\left(\Xi_{j\left(x_{k}\right)}-\Xi\right) \in \operatorname{Pic}^{0} P$.

Lemma 3.1. The sheaf $\mathcal{R}^{0}$ is locally free of rank 1.

Proof. The resolution (14) shows that $\mathcal{R}^{0}$ is a 2 -syzygy of rank 1 . If $\mathcal{R}^{0}$ were not invertible, this would contradict the Evans-Griffith theorem on syzygies [EG, Cor. $1.7]$.

3.2. Duality and the sheaf $\mathcal{R}^{1}$. As explained in 1.2 , we have the isomorphisms of functors,

$$
\mathbf{R} \Delta_{P} \circ \mathbf{R} \mathscr{S}_{P} \cong[g-1] \circ(-1)^{*} \mathbf{R} \mathscr{S}_{P} \circ \mathbf{R} \Delta_{P}
$$

and

$$
\mathbf{R} \Delta_{P} \circ j_{*} \cong[2-g] \circ j_{*} \circ \mathbf{R} \Delta_{\widetilde{C}} .
$$

All together applied to the element $L^{-}$gives

$$
\begin{aligned}
(-1)^{*} \mathbf{R} \mathcal{H o m}\left(\mathbf{R} \mathscr{S}_{P}\left(j_{*} \sigma^{*} L^{-}\right), \mathcal{O}_{P}\right) & \cong \mathbf{R} \mathscr{S}_{P}\left(j_{*}\left(\mathbf{R} \mathcal{H o m}_{\widetilde{C}}\left(\sigma^{*} L^{-}, \omega_{\widetilde{C}}\right)\right)\right)[1] \\
& \cong \mathbf{R} \mathscr{S}_{P}\left(j_{*} L^{-}\right)[1]
\end{aligned}
$$

(observe that $\left.L^{-} \otimes \sigma^{*} L^{-} \cong \pi^{*} N m_{\pi}\left(L^{-}\right) \cong \pi^{*} \omega_{C} \cong \omega_{\widetilde{C}}\right)$.

Lemma 3.2. Given any $L^{-} \in P^{-}$, consider

$$
\begin{aligned}
\mathcal{R}^{0}=R^{0} \mathscr{S}_{P}\left(j_{*} L^{-}\right), & \mathcal{R}^{1}=R^{1} \mathscr{S}_{P}\left(j_{*} L^{-}\right) \text {and } \\
\mathcal{R}_{\sigma}^{0}=(-1)^{*} R^{0} \mathscr{S}_{P}\left(j_{*} \sigma^{*} L^{-}\right), & \mathcal{R}_{\sigma}^{1}=(-1)^{*} R^{1} \mathscr{S}_{P}\left(j_{*} \sigma^{*} L^{-}\right) .
\end{aligned}
$$

Then,

$$
\begin{aligned}
& \mathcal{H o m}\left(\mathcal{R}^{1}, \mathcal{O}_{P}\right) \cong \mathcal{R}_{\sigma}^{0} \\
& \mathcal{E} x t^{1}\left(\mathcal{R}^{1}, \mathcal{O}_{P}\right)=0 \\
& \mathcal{E} x t^{q}\left(\mathcal{R}^{1}, \mathcal{O}_{P}\right)=0 \text { for all } q>3, \text { and } \\
& 0 \rightarrow \mathcal{R}^{1} \rightarrow \mathcal{H o m}\left(\mathcal{H o m}\left(\mathcal{R}^{1}, \mathcal{O}_{P}\right), \mathcal{O}_{P}\right) \rightarrow \mathcal{E} x t^{2}\left(\mathcal{R}_{\sigma}^{1}, \mathcal{O}_{P}\right) \rightarrow 0 .
\end{aligned}
$$

In particular, $\mathcal{R}^{1}$ is torsion-free.

Proof. The isomorphism (16) in the derived category of sheaves, induces the following spectral sequence,

$$
E_{2}^{i, j}:=\mathcal{E} x t^{i}\left((-1)^{*} R^{-j} \mathscr{S}_{P}\left(j_{*} \sigma^{*} L^{-}\right), \mathcal{O}_{P}\right) \Rightarrow R^{i+j+1} \mathscr{S}_{P}\left(j_{*} L^{-}\right) .
$$

This is a fourth quadrant spectral sequence with only 2 rows and the only non-zero element in the row $j=0$ is $E_{2}^{0,0}$ since $\mathcal{R}_{\sigma}^{0}$ is locally free. It is easy to deduce from that

$$
\begin{aligned}
& \mathcal{R}^{0} \cong \mathcal{H o m}\left(\mathcal{R}_{\sigma}^{1}, \mathcal{O}_{P}\right), \\
0 \rightarrow \mathcal{E} x t^{1}\left(\mathcal{R}_{\sigma}^{1}, \mathcal{O}_{P}\right) \rightarrow & \mathcal{R}^{1} \rightarrow \mathcal{H o m}\left(\mathcal{R}_{\sigma}^{0}, \mathcal{O}_{P}\right) \rightarrow \mathcal{E} x t^{2}\left(\mathcal{R}_{\sigma}^{1}, \mathcal{O}_{P}\right) \rightarrow 0, \\
& \mathcal{E} x t^{q}\left(\mathcal{R}_{\sigma}^{1}, \mathcal{O}_{P}\right)=0 \text { for all } q \geq 3
\end{aligned}
$$


Since $(-1)^{*}$ and $\sigma^{*}$ are involutions in $\operatorname{Pic}(P)$, we also have the same statements substituting $\mathcal{R}^{i}$ by $\mathcal{R}_{\sigma}^{i}$ and vice versa. Observe that the middle morphism $\mathcal{R}^{1} \rightarrow$ $\mathcal{H o m}\left(\mathcal{R}_{\sigma}^{0}, \mathcal{O}_{P}\right) \cong \mathcal{H o m}\left(\mathcal{H o m}\left(\mathcal{R}^{1}, \mathcal{O}_{P}\right), \mathcal{O}_{P}\right)$ is the natural map from any sheaf to its double dual. Then we use the following result on coherent sheaves (see for instance [HL, Prop. 1.1.10]): for a sheaf $E$ on a smooth variety $X$, the following are equivalent:

(i) $\operatorname{codim}\left(\operatorname{supp}\left(\mathcal{E} x t^{q}\left(E, \omega_{X}\right)\right)\right) \geq q+1, \quad \forall q>0$.

(ii) $E \hookrightarrow \mathcal{H o m}\left(\mathcal{H o m}\left(E, \omega_{X}\right), \omega_{X}\right)$.

In our case $X=P, \omega_{X}=\mathcal{O}_{P}$ and $E=\mathcal{R}_{\sigma}^{1}$. We have seen that $\mathcal{E}_{x} t^{q}\left(\mathcal{R}_{\sigma}^{1}, \mathcal{O}_{P}\right)=$ $0, \forall q \geq 3$. Moreover $\mathcal{R}_{\sigma}^{1}$ is locally free out of $V^{2}$; hence, $\operatorname{supp}\left(\mathcal{E} x t^{q}\left(\mathcal{R}_{\sigma}^{1}, \mathcal{O}_{P}\right)\right) \subset V^{2}$. Since $V^{2}$ has codimension 3, condition (i) is fulfilled. Condition (ii) implemented in (18) completes the proof of the lemma.

3.3. The sheaf $\mathcal{R}^{0}$. In view of Lemma 3.1, $\mathcal{R}^{0}$ corresponds to a Cartier divisor. We want to determine this divisor modulo algebraic equivalence. In order to do that, we will consider Chern classes as a cycles modulo algebraic equivalence. We identify $\mathrm{Pic}^{0} P$ with $P$ as ppav via the isomorphism induced by $\Xi$.

Going back to (14) and (15), since $c_{1}\left(\mathcal{G}^{0}\right)=\Sigma c_{1}\left(\mathcal{L}_{j\left(x_{k}\right)}\right)=0$, we get

$$
c_{1}\left(\mathcal{R}^{0}\right)-c_{1}\left(\mathcal{R}^{1}\right)=c_{1}\left(\mathcal{E}^{0}\right) .
$$

The isomorphism (17) allows us to compare $c_{1}\left(\mathcal{R}^{1}\right)$ and $c_{1}\left(\mathcal{R}^{0}\right)$.

Lemma 3.3. One has $-c_{1}\left(\mathcal{R}^{1}\right)=c_{1}\left(\mathcal{R}^{0}\right)$.

Proof. The sheaf $\mathcal{R}^{1}$ is invertible on the complement of a closed subset of codimension 3 , therefore by (17),

$$
-c_{1}\left(\mathcal{R}^{1}\right)=c_{1}\left(\mathcal{R}_{\sigma}^{0}\right)=c_{1}\left((-1)^{*} R^{0} \mathscr{S}_{P}\left(j_{*} \sigma^{*} L^{-}\right)\right) .
$$

Since in codimension 1 algebraic and homological equivalence coincide, we have that

$$
c_{1}\left((-1)^{*} R^{0} \mathscr{S}_{P}\left(j_{*} \sigma^{*} L^{-}\right)\right)=c_{1}\left(R^{0} \mathscr{S}_{P}\left(j_{*} \sigma^{*} L^{-}\right)\right) .
$$

Now we want to see that $c_{1}\left(R^{0} \mathscr{S}_{P}\left(j_{*} \sigma^{*} L^{-}\right)\right)=c_{1}\left(\mathcal{R}^{0}\right)$.

More precisely, we will see that the algebraic equivalence class of $c_{1}\left(R^{0} \mathscr{S}_{P}\left(j_{*} L^{-}\right)\right)$ does not depend on $L^{-} \in P^{-}$. Let $L_{1}^{-} \in P^{-}$be another element. Put $L_{1}^{-} \otimes$ $\left(L^{-}\right)^{-1}=j^{*} \alpha$, for some $\alpha \in \operatorname{Pic}^{0}(P)$. Then

$$
\mathbf{R} \mathscr{S}_{P}\left(j_{*} L_{1}^{-}\right) \cong \mathbf{R} \mathscr{S}_{P}\left(j_{*} L^{-} \otimes \alpha\right) \cong t_{\alpha}^{*} \mathbf{R} \mathscr{S}_{P}\left(j_{*} L_{1}^{-}\right) \text {. }
$$

The last isomorphism is a consequence of the commutation between translation isomorphisms and tensoring with elements of $\operatorname{Pic}^{0}(P)$ proved by Mukai [Mu1, (3.1)]. Now take 0 -cohomology and $c_{1}$.

Hence

$$
2 c_{1}\left(\mathcal{R}^{0}\right)=c_{1}\left(\mathcal{E}^{0}\right) .
$$

We now use [Mu2, Cor. 1.18] for a $\operatorname{WIT}\left(i_{0}\right)$-sheaf $\mathcal{F}$,

$$
\operatorname{ch}_{i}\left(R^{i_{0}} \mathscr{S}(\mathcal{F})\right)=(-1)^{i+i_{0}} P D\left(\operatorname{ch}_{a-i}(\mathcal{F})\right),
$$

where $a$ is the dimension of the abelian variety and $P D$ stands for the Poincaré duality isomorphism.

Since $\mathcal{E}^{0}=R^{0} \mathscr{S}_{P}\left(j_{*}\left(L^{-}(E)\right)\right)$ and $j_{*}\left(L^{-}(E)\right)$ is $\operatorname{WIT}(0)$,

$$
c_{1}\left(\mathcal{E}^{0}\right)=\operatorname{ch}_{1}\left(R^{0} \mathscr{S}\left(j_{*}\left(L^{-}(E)\right)\right)\right)=-P D\left(\operatorname{ch}_{g-2}\left(j_{*}\left(L^{-}(E)\right)\right)\right) \text {. }
$$


The Grothendieck-Riemann-Roch formula says

$$
\begin{aligned}
\operatorname{ch}\left(j_{*}\left(L^{-}(E)\right) \cdot \operatorname{todd}\left(T_{P}\right)\right) & =j_{*}\left(\operatorname{ch}_{\widetilde{C}}\left(L^{-}(E)\right) \cdot \operatorname{todd}\left(T_{\widetilde{C}}\right)\right) \\
& =j_{*}\left(\left(1+L^{-}(E)\right) \cdot\left(1-K_{\widetilde{C}}\right)\right) \\
& =[\widetilde{C}]+\operatorname{deg}\left(L^{-}(E)-K_{\widetilde{C}}\right) \\
& =2 \frac{[\Xi]^{g-2}}{(g-2) !}+m-2 g+2 .
\end{aligned}
$$

Hence

$$
c_{1}\left(\mathcal{E}^{0}\right)=-2 P D\left(\frac{[\Xi]^{g-2}}{(g-2) !}\right)=-2[\Xi] .
$$

Lemma 3.4. For some $\lambda \in P$ there is an isomorphism,

$$
\mathcal{R}^{0} \cong \mathcal{O}_{P}\left(-\Xi_{\lambda}\right) \text {. }
$$

Proof. Implementing (20) in (19) we reach to

$$
-2 c_{1}\left(\mathcal{R}^{0}\right)=2[\Xi]
$$

which easily implies the statement.

3.4. Application of Mukai's theorem. The main theorem in [Mu1, Thm. 2.2] says that

$$
\mathbf{R} \mathscr{S}_{P} \circ \mathbf{R} \mathscr{S}_{P} \cong(-1)^{*}[-(g-1)] .
$$

This gives the spectral sequence,

$$
E_{2}^{i j}:=R^{i} \mathscr{S}_{P}\left(R^{j} \mathscr{S}_{P}\left(j_{*} L^{-}\right)\right) \Longrightarrow H^{i+j}:= \begin{cases}(-1)^{*} j_{*} L^{-} & \text {if } i+j=g-1, \\ 0 & \text { otherwise. }\end{cases}
$$

Observe that $E_{2}^{i j}=0$ if $i \geq g$ or $j \geq 2$. Hence everything vanishes except two rows. Putting $R^{i j}:=E_{2}^{i j}$ we obtain the following information:

(a) Observe that, by Lemma 3.4. $R^{g-1,0}=R^{g-1} \mathscr{S}_{P}\left(\mathcal{O}_{P}\left(-\Xi_{\lambda}\right)\right) \cong(-1)^{*} \mathcal{O}_{P}\left(\Xi_{\lambda}\right)$ is the only non-zero element for $j=0$. Then, all the differentials from $R^{i, 1}$ vanish unless $i=g-3$. If $i+j \neq g-1$, then $E_{\infty}^{i, j}=0$. Hence, the only non-zero elements for $j=1$ are $R^{g-3,1}$ and $R^{g-2,1}$.

(b) There is an exact sequence,

$$
0 \rightarrow R^{g-3,1} \rightarrow(-1)^{*} \mathcal{O}_{P}\left(\Xi_{\lambda}\right) \rightarrow(-1)^{*} j_{*} L^{-} \rightarrow R^{g-2,1} \rightarrow 0 .
$$

In particular $(-1)^{*} R^{g-3,1}$ is an ideal sheaf $\mathcal{I}_{Z}$ twisted with $\Xi_{\lambda}$. Therefore, (21) provides a short exact sequence on $\widetilde{C}$,

$$
0 \rightarrow(-1)^{*} \mathcal{O}_{Z}\left(\Xi_{\lambda}\right) \rightarrow(-1)^{*} j_{*} L^{-} \rightarrow R^{g-2,1} \rightarrow 0 .
$$

Hence $Z=\widetilde{C}$ and $(-1)^{*} R^{g-3,1} \cong \mathcal{I}_{\widetilde{C}}\left(\Xi_{\lambda}\right)$. Moreover $\mathcal{O}_{\widetilde{C}}\left(\Xi_{\lambda}\right)$ has degree $2 g-2=$ $\operatorname{deg} L^{-}$, so $R^{g-2,1}=0$.

Corollary 3.5. The sheaf $\mathcal{R}^{1}$ is $W I T(g-3)$ and its Fourier-Mukai transform is $\mathcal{I}_{\widetilde{C}}\left(\Xi_{\lambda}\right)$.

Remark 3.6. Since the projectivization of the sheaf $\mathbf{R} \mathscr{S}_{J \widetilde{C}}\left(i_{*}\left(L^{-}\right)\right)$is $\widetilde{C}^{(2 g-2)}$, by (3) the projectivization of $\mathcal{R}^{1}$ is $X^{-}$. Then one could try to recover directly $\mathcal{R}^{1}$ from $X^{-}$imitating the argument in $[\mathrm{Na}, \S 4]$. In this case, $\mathcal{R}^{1}$ is locally free on 
$P \backslash V^{2} \stackrel{i}{\hookrightarrow} P$, but it is easy to see that $i_{*} i^{*} \mathcal{R}^{1} \neq \mathcal{R}^{1}$, and this fact invalidates this direct strategy.

3.5. The scheme-theoretic equality $T\left(V^{2}\right)=\widetilde{C}$. The equality $T(\widetilde{C})=V^{2}$ obtained in the last section combined with (2) gives the following corollary.

Corollary 3.7. There is an isomorphism of invertible sheaves on $V^{2}$,

$$
\mathcal{H o m}\left(\mathbf{R} \mathscr{S}_{P}\left(\mathcal{I}_{\widetilde{C}}\left(\Xi_{\lambda}\right)\right), \mathcal{O}_{P}\right) \cong \mathcal{O}_{V^{2}}\left(\Xi_{\lambda}\right) \text {. }
$$

Isomorphism (22) in combination with Corollary 3.5 allows us to prove the following result.

Proposition 3.8. We have

$$
(-1)^{*} \mathcal{E} x t^{2}\left(\mathcal{R}^{1}, \mathcal{O}_{P}\right) \cong \mathcal{O}_{V^{2}}\left(\Xi_{\lambda}\right) .
$$

In particular, by Corollary $\left[3.5, \mathcal{R}^{1} \cong \mathcal{I}_{V^{2}}\left(\Xi_{\lambda}\right)\right.$.

Proof. By Corollary 3.5 we can replace in (22) the sheaf $\mathcal{I}_{\widetilde{C}}\left(\Xi_{\lambda}\right)$ by $R^{g-3} \mathscr{S}_{P}\left(\mathcal{R}^{1}\right)=$ $\mathbf{R} \mathscr{S}_{P}\left(\mathcal{R}^{1}\right)[g-3]$. So,

$$
\mathcal{O}_{V^{2}}\left(\Xi_{\lambda}\right) \cong \mathcal{H o m}\left((-1)^{*} \mathcal{R}^{1}[1-g+g-3], \mathcal{O}_{P}\right) \cong(-1)^{*} \mathcal{E} x t^{2}\left(\mathcal{R}^{1}, \mathcal{O}_{P}\right) .
$$

Corollary 3.9. The scheme-theoretic equality $T\left(V^{2}\right)=\widetilde{C}$ holds.

Proof. In Proposition 3.8 we have obtained an expression of the ideal of $V^{2}$ (tensored with the Theta divisor) in terms of $\mathcal{R}^{1}$. By replacing in the definition of $T\left(V^{2}\right)$ we get,

$$
\begin{aligned}
T\left(V^{2}\right) & =\operatorname{supp}\left(\mathcal{H o m}\left(\mathbf{R} \mathscr{S}_{P}\left(\mathcal{R}^{1}\right), \mathcal{O}_{P}\right)\right. \\
& =\operatorname{supp}\left(\mathcal{H o m}\left(\mathcal{I}_{\widetilde{C}}\left(\Xi_{\lambda}\right)[(g-3)], \mathcal{O}_{P}\right)\right) \\
& =\operatorname{supp}\left(\mathcal{E} x t^{g-3}\left(\mathcal{I}_{\widetilde{C}}\left(\Xi_{\lambda}\right), \mathcal{O}_{P}\right)\right) \\
& =\operatorname{supp}\left(\mathcal{E} x t^{g-2}\left(\mathcal{O}_{\widetilde{C}}\left(\Xi_{\lambda}\right), \mathcal{O}_{P}\right)\right) \\
& =\widetilde{C} .
\end{aligned}
$$

The last equality is standard and can be obtained, for example, by noticing that $\left.\mathcal{E}_{x} t^{g-2}\left(\mathcal{O}_{\widetilde{C}}\left(\Xi_{\lambda}\right), \mathcal{O}_{P}\right)\right)$ is the dual sheaf of $\mathcal{O}_{\widetilde{C}}\left(\Xi_{\lambda}\right)$ in $P$ and that duality commutes with $j_{*}$ (cf. [HL, pg. 5]).

Remark 3.10. The scheme-theoretic equalities $T(T(\widetilde{C}))=\widetilde{C}$ and $T\left(T\left(V^{2}\right)\right)=V^{2}$ tell us that $\widetilde{C}$ and $V^{2}$ are scheme-theoretically intersections of translates of $\Xi$.

\section{Reduceness of $V^{2}$ And the proof of Theorem B}

\section{1. $V^{2}$ is reduced.}

Lemma 4.1. For any étale double cover $\widetilde{C} \rightarrow C$, such that $C$ is a non-hyperelliptic curve of genus $g>4, V^{2}$ is reduced, pure-dimensional and Cohen-Macaulay.

Proof. Step 1. If $\operatorname{dim} V^{2}>0$, then $V^{2} \backslash V^{4}$ is generically smooth: Let $L \in V^{2} \backslash V^{4}$, so $h^{0}(\widetilde{C}, L)=3$. Suppose that $V^{2}$ is singular at $L$, so

$$
\operatorname{dim} T_{L} V^{2}>g-4 .
$$


The Zariski tangent space $T_{L} V^{2}$ is given as the orthogonal complement to the image of the map (see [W1, (1.9)]),

$$
v_{0}: \bigwedge^{2} H^{0}(\widetilde{C}, L) \rightarrow H^{0}\left(\widetilde{C}, \omega_{\widetilde{C}}\right)^{-}
$$

defined by $v_{0}\left(s_{i} \wedge s_{j}\right)=s_{i} \sigma^{*} s_{j}-s_{j} \sigma^{*} s_{i}$.

The inequality (23) is equivalent to $\operatorname{dim}\left(\operatorname{im} v_{0}\right)<3$. On the other hand, all the forms in $\bigwedge^{2} H^{0}(\widetilde{C}, L)$ are decomposable and $\operatorname{dim} \bigwedge^{2} H^{0}(\widetilde{C}, L)=$ 3 , so there is a decomposable form $s_{i} \wedge s_{j}$ in ker $v_{0}$. This means that $s_{i} \sigma^{*} s_{j}-s_{j} \sigma^{*} s_{i}=0$, or in other words, that $\frac{s_{j}}{s_{i}}$ defines a rational function $h$ on $C$. This induces a map $C \rightarrow \mathbb{P}^{1}$, so there exists a line bundle $M$, with $h^{0}(M) \geq 2$, such that $L \cong \pi^{*} M \otimes \mathscr{O}_{\widetilde{C}}(B)$ and $B$ is an effective divisor (the maximal common divisor between $\left(s_{i}\right)_{0}$ and $\left.\left(s_{j}\right)_{0}\right)$.

Observe that $N m(B) \in\left|K_{C} \otimes M^{\otimes-2}\right|$ and for any $L \in V^{2} \backslash V^{4}$ such that $V^{2}$ is singular at $L$, we obtain a pair $(M, B)$. The family of such pairs $(M, B)$ is a finite cover of the set of pairs $(M, F)$ where:

- $M$ is an invertible sheaf on $C$ of degree $d \geq 2$ such that $h^{0}(C, M) \geq 2$,

- $F$ is an effective divisor on $C$ of degree $2 g-2-2 d \geq 0$, such that $F \in\left|K_{C} \otimes M^{\otimes-2}\right|$.

By Martens' theorem applied to the non-hyperelliptic curve $C$ (see ACGH, p. 192]), the dimension of the above family of line bundles $M$ is bounded above by

$$
\operatorname{dim}\left(W_{d}^{1}\right) \leq d-3 .
$$

Fixing a line bundle $M$ as above, the dimension of possible $F$ satisfying the second condition is bounded by Clifford's theorem (e.g. ACGH, p. 107]),

$$
h^{0}\left(K_{C} \otimes M^{\otimes-2}\right)-1 \leq g-1-d .
$$

Moreover, equality holds if and only if $M$ is a two torsion point or a thetacharacteristic. The first possibility is excluded since $h^{0}(C, M) \geq 2$. If $M$ is a theta-characteristic, then $F=0$ and there is only a finite number of pairs $(M, F)$. Hence, a finite number of singular points on $V^{2} \backslash V^{4}$.

When equality does not hold, i.e. $h^{0}\left(K_{C} \otimes M^{\otimes-2}\right)-1 \leq g-2-d$, together with inequality (24), we get that the dimension $m$ of our family of pairs $(M, F)$ is bounded above by $m \leq d-3+g-2-d=g-5$. So $V^{2} \backslash V^{4}$ is singular at most in codimension 4 in the Prym variety.

In both cases we have that, if $V^{2}$ is positive dimensional, then $V^{2} \backslash V^{4}$ is generically smooth.

Step 2. $V^{2} \backslash V^{4}$ has non-empty intersection with every component of $V^{2}$ : Suppose that a component of $u^{-1} V^{2}$ is entirely contained in $u^{-1} V^{4}$. Let $D$ be a general point of a component of $u^{-1} V^{2}$ and assume that $D \in u^{-1} V^{4}$. Then, for every $p, q \in \widetilde{C}, h^{0}(D-p-q)>0$ and if they are not base points of $|D|$, then $h^{0}(D-p-q)=h^{0}(D)-2>0$. Take $E \in|D-p-q|$. By the parity trick of Mumford [M1, p. 188, Step II] $h^{0}(D-p-q+\sigma p+\sigma q)=h^{0}(D)-2$ and by the generality of $p$ and $q, E+\sigma p+\sigma q$ belongs to the same component of $u^{-1} V^{2}$ as $D$, which contradicts by semicontinuity the generality of $D$. 
Step 3. $V^{2}$ is reduced: By the previous steps, to show that $V^{2}$ is reduced is enough to see that it has no embedded components. If $V^{2}$ is CohenMacaulay this is straightforward. $V^{2}$ is Cohen-Macaulay and equidimensional if, and only if, $\mathcal{E} x t^{k}\left(\mathcal{O}_{V^{2}}, \mathcal{O}_{P}\right) \neq 0 \Leftrightarrow k=3$. Since $\mathcal{R}^{1} \cong \mathcal{I}_{V^{2}}\left(\Xi_{\lambda}\right)$, by Proposition 3.8, this follows from Lemma 3.2 .

Remark 4.2. When $C$ is a generic curve of genus $4, V^{2}$ consists of two different points. However it could happen that $V^{2}$ is not reduced for some coverings. In that case, the curve $C$ would have a unique $g_{3}^{1}$ complete linear series.

Remark 4.3. The authors have been informed by A. Höring that he has obtained this result in [H2, Thm. 1.1] for $g \geq 6$. In fact, he also studies when $V^{2}$ is reducible. One of these cases is the intermediate Jacobian of the cubic threefold that we consider in the last section of this paper. Since $V^{2}$ has the expected dimension, the fact that $V^{2}$ is Cohen-Macaulay (shown in Step 3) is also proved in [D2, (6.2) 1)]. We keep our proof here for the convenience of the reader since it is a rather direct consequence of our computations.

In particular, $V^{2}$ is pure-dimensional and, as a corollary of [dCP, Thm. 9], we have the following.

Corollary 4.4. The cohomological class of $V^{2}$ is

$$
\left[V^{2}\right]=2 \frac{\Xi^{3}}{6}
$$

4.2. Proof of Theorem B, Now one can easily prove Theorem[B] Let $\mu: \widetilde{X^{-}} \rightarrow$ $X^{-}$be a desingularization of $X^{-}$. The composition of $\mu$ with $X^{-} \rightarrow P$ provides a birational morphism from the smooth variety $\widetilde{X^{-}}$to an abelian variety $P$, which is necessarily the Albanese map of $\widetilde{X^{-}}$. Thus, the Albanese map of any desingularization of $X^{-}$factorizes through $X^{-}$and gives a well-defined map $X^{-} \rightarrow P$ (up to translation). The locus where the fibers of this map have positive dimension (considered with its reduced structure) is $V^{2}$. Then, we can also recover the divisor $\Xi$ (up to translation), since there is only one principal polarization such that the cohomology class of $V^{2}$ is $2 \frac{\Xi^{3}}{6}$ (cf. [Ra, pp. 288-289]). Thus, we can consider the theta-dual of $V^{2}, \widetilde{C}=T\left(V^{2}\right)$ and recover the curve $\widetilde{C}$ naturally embedded in the Prym variety. Now, a well-known argument of Welters [W3, (2.2), p. 96] allows us to recover the involution $\sigma^{*}$ in $\operatorname{Pic}^{0} \widetilde{C}$. Since $\widetilde{C}$ is non-hyperelliptic, by the strong Torelli theorem, the involution $\sigma$ in $\widetilde{C}$ is recovered.

\section{5. $V^{2}$ IN THE INTERMEDiATE JACOBIANS OF CUBIC THREEFOLS}

The aim of this section is to illustrate Theorem A in a very well-known context: the intermediate Jacobian $J$ of a smooth cubic threefold $V$, since $J$ is isomorphic (as ppav) to a Prym variety of dimension 5. We closely follow the notation and results of [B1] and CG. Let us fix some terminology and recall some basic facts. Let $F$ be the Fano surface of the lines contained in $V$. Fixing a point in $F$, the Albanese map gives an embedding $F \hookrightarrow A l b(F) \cong J$. Let $t \in F$ be general and denote by $C_{t}$ 
the (smooth irreducible of genus 11) curve of the lines of $F$ intersecting $t$. Observe that this curve comes equipped with two natural structures:

a) An involution $\sigma$ : if $r \in C_{t}$, the 2-plane $t \vee r$ intersects $V$ in 3 lines, $t, r$ and $\sigma r$. The quotient $C_{t} / \sigma=: D_{t}$ is a smooth plane quintic. It turns out that $P\left(C_{t}, D_{t}\right) \cong J$.

b) A complete $g_{5}^{1}$ attached to the map $C_{t} \rightarrow t$ mapping $r$ to the intersection point $r \cap t$. Denote by $L \in \operatorname{Pic}^{5}\left(C_{t}\right)$ the corresponding line bundle. Beauville proves $[\mathrm{B} 2, \S 4 . \mathrm{C}]$ that there are only 2 such linear series with norm $\mathcal{O}(1)$ on $C_{t}: L$ and $\sigma L$.

Following Beauville, we denote by $D(r) \in C_{t}^{(5)}$ the divisor $C_{t} \cap C_{r}$, i.e. the five lines that intersect both $t$ and $r$. Observe that if $r$ intersects $t$, then $\mathcal{O}_{C_{t}}(D(r)) \cong$ $L(\sigma r-r)$. All these divisors have norm in the linear system $|\mathcal{O}(1)|$ of $D_{t}$. So it is natural to consider the special subvarieties attached to $|\mathcal{O}(1)|$ by taking the following fiber product diagram,

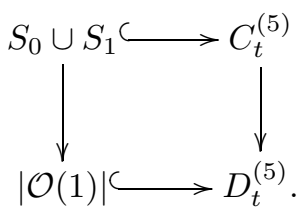

The divisors $D(r)$ belong to one of these components, say $S_{0}$. Then the map $F \backslash\{t\} \rightarrow S_{0}$ extends to the blow-up $F_{t}$ of $F$ at $t$, and $F_{t} \rightarrow S_{0}$ is an isomorphism (cf. [B1, Prop. 3]). The exceptional divisor maps to $|\sigma L|$. The composition $F_{t} \rightarrow S_{0} \longrightarrow P \cong J$ factors through the Albanese map $F \hookrightarrow J$.

Lemma 5.1. With the same notation:

(a) $V^{3}=\left\{\pi^{*}(\mathcal{O}(1))\right\}=\operatorname{Sing}(\Xi), V^{4}=\emptyset$.

(b) $h^{0}\left(C_{t}, L^{\otimes 2}\right)=h^{0}\left(C_{t}, \sigma L^{\otimes 2}\right)=3$.

(c) For all $r \in F \backslash\{t\}, h^{0}\left(C_{t}, \sigma L(D(r))\right)=h^{0}\left(C_{t}, L(\sigma D(r))\right)=3$.

Proof. The statement on $V^{3}$ is proved in [B1, Prop. 2] (in fact it implies that $J$ is not a product of Jacobians and therefore $V$ is not rational). If $V^{4}$ were non-empty, then by twisting with $r-\sigma r$ we will produce infinite elements in $V^{3}$. To prove (b) observe that $L^{\otimes 2}$ has norm $\mathcal{O}(2) \cong \omega_{D_{t}}$ and it is obtained from $\pi^{*}(\mathcal{O}(1))=L \otimes \sigma L \in P^{+}$by twisting with 5 elements of the form $x-\sigma x$, hence it belongs to $P^{-}$. Since $2=h^{0}\left(C_{t}, L\right) \leq h^{0}\left(C_{t}, L^{\otimes 2}\right)$ and $V^{4}$ is empty, the statement follows. By applying $\sigma$, the same is true for $\sigma L^{\otimes 2}$. Finally (c) is a consequence of (b) since $\sigma L(D(r))$ specializes to $\sigma L^{\otimes 2}$.

From now on we think of $F$ as a surface embedded in the canonical model of $P\left(C_{t}, D_{t}\right) \cong J$ :

$$
P^{+}=\left\{M \in \operatorname{Pic}^{10}\left(C_{t}\right) \mid N m_{\pi}(M) \cong \mathcal{O}_{D_{t}}(1), h^{0}(M) \text { even }\right\}
$$

by means of the map $r \mapsto \mathcal{O}_{C_{t}}(D(r)) \otimes L$. Put $F_{a}:=a+F=t_{-a}^{*} F$ and $(\sigma F)_{a}:=$ $a+\sigma F=t_{-a}^{*}(\sigma F)$. By using the previous lemma and comparing the cohomology class of $V^{2}$ with that of $F$ the following equality in $P^{-}$is straightforward.

Corollary 5.2. We have $V^{2}=F_{\sigma L \otimes L^{-1}} \cup(\sigma F)_{L \otimes \sigma L^{-1}}$. 
The main theorem of [H1] combined with the results in [PP, §8.1] imply that

$$
\begin{aligned}
& T\left(F_{\sigma L \otimes L^{-1}}\right)=(\sigma F)_{\sigma\left(L^{-2}\right)}=(-1)^{*}\left(F_{L^{-2}}\right) \subset P^{\prime}, \\
& T\left((\sigma F)_{L \otimes \sigma L^{-1}}\right)=F_{L^{-2}} \subset P^{\prime} .
\end{aligned}
$$

Hence

$$
T\left(V^{2}\right)=F_{L^{-2}} \cap(-1)^{*}\left(F_{L^{-2}}\right) .
$$

The last piece that completes the picture is the observation that

$$
\mathcal{O}_{C_{t}}(D(r)+D(\sigma r)) \cong L^{\otimes 2}
$$

for any $r$ intersecting $t$, i.e. for every $r \in C_{t}$. This says that

$$
\begin{aligned}
C_{t} & \hookrightarrow T\left(V^{2}\right) \\
r & \mapsto L^{-1}(D(r)) .
\end{aligned}
$$

Theorem $\mathrm{A}$ shows that this inclusion $C_{t} \subset T\left(V^{2}\right)$ is in fact an equality of schemes.

Summarizing we get the following.

Proposition 5.3. With the notation above, $T\left(V^{2}\right)=F_{L^{-2}} \cap(-1)^{*}\left(F_{L^{-2}}\right)=C_{t}$.

\section{ACKNOWLEDGMENTS}

We wish to thank Andreas Höring for sharing his work $\mathrm{H} 2$ with us. We also thank Miguel Ángel Barja for many valuable discussions, especially while carrying out the first author's Ph.D. thesis. The second author is grateful to Alberto Collino and Gian Pietro Pirola for stimulating conversations on the geometry of the cubic threefolds. Finally, we thank the referee for many suggestions that improved the exposition of the paper.

\section{REFERENCES}

[ACGH] E. Arbarello, M. Cornalba, P.A. Griffiths, J. Harris, Geometry of algebraic curves. Vol. I. Grundlehren der Mathematischen Wissenschaften, 267. Springer-Verlag, New York, 1985. MR770932 (86h:14019)

[B1] A. BeAuville, Les singularités du diviseur $\Theta$ de la jacobienne intermédiaire de l'hypersurface cubique dans $\mathbb{P}^{4}$, Algebraic threefolds (Varenna, 1981), Lecture Notes in Math., 947, Springer, Berlin (1982), pp. 190-208. MR672617 (84c:14030)

[B2] A. Beauville, Sous-variétés spéciales des variétés de Prym, Compositio math., 45 (1981), pp. 357-383. MR656611 (83f:14025)

[CLV] S. Casalaina-Martin, M. Lahoz, F. Viviani, Cohomological support loci for Abel-Prym curves, Le Matematiche, 63 (2008), pp. 205-222. MR2512518(2010a:14060)

[CG] C.H. Clemens. P.A. Griffiths, The intermediate Jacobian of the cubic threefold, Ann. of Math. 95 (1972), pp. 281-356. MR0302652(46:1796)

[D1] O. Debarre, Sur les variétés de Prym des courbes tétragonales, Ann. Sci. École Norm. Sup., 21 (1988), pp. 545-559. MR982333(90b:14034)

[D2] O. Debarre, Théorèmes de Lefschetz pour les lieux de dégénérescence, Bull. Soc. Math. France, 128 (2000), pp. 283-308. MR.1772444 (2002b:14051)

[Do] R. Donagi, The tetragonal construction, Bull. Amer. Math. Soc. 4 (1981), pp. 181-185. MR.598683(82a:14009)

[dCP] C. De Concini, P. Pragacz, On the class of Brill-Noether loci for Prym varieties, Math. Ann., 302 (1995), pp. 687-697. MR.1343645 (97e:14040)

[EG] E.G. Evans, P. Griffith, The syzygy problem, Annals of Math., 114 (1981), pp. 323333. MR632842 (83i:13006)

[GL] M.G. Gulbrandsen, M. Lahoz, Finite subschemes of abelian varieties and the Schottky problem, arXiv:1004.1495. To appear in Ann. Inst. Fourier (Grenoble).

[H1] A. Höring, M-regularity of the Fano surface, C. R. Math. Acad. Sci. Paris 344 (2007), 691-696. MR2334677 (2008g:14063) 
[H2] A. HöRInG, Geometry of Brill-Noether loci on Prym varieties, Preprint 2011. arXiv:1103.1053.

[Hu] D. Huybrechts, Fourier-Mukai transforms in algebraic geometry, Oxford Mathematical Monographs, 2006. MR2244106 (2007f:14013)

[HL] D. Huybrechts, M. LeHN, The geometry of moduli spaces of sheaves, Aspects of Mathematics E., V. 31, Friedr. Vieweg \& Sohn, Braunschweig, 1997. MR1450870 (98g:14012)

[Iz] E. IzADI, The geometric structure of $\mathcal{A}_{4}$, the structure of the Prym map, double solids and $\Gamma_{00}$-divisors, J. Reine Angew. Math. 462 (1995), 93-158. MR1329904 (96d:14042)

[IL] E. Izadi, H. Lange, Counter-examples of high Clifford index to Prym-Torelli. Preprint 2010. arXiv:1001.3610.

[La] M. LAhoz, Theta-duality in Abelian Varieties and the Bicanonical Map of Irregular Varieties, Ph.D. Thesis, Universitat Politècnica de Catalunya, May 2010.

[M1] D. Mumford, Theta characteristics of an algebraic curve, Ann. Sci. École Norm. Sup. 4 (1971), 181-192. MR0292836(45:1918)

[M2] D. Mumford, Prym Varieties I, Contributions to Analysis, 1974, Ahlfors, L., Academic Press, New York. MR0379510 (52:415)

[Mu1] S. MukaI, Duality between $D(X)$ and $D(\hat{X})$ with its application to Picard sheaves, Nagoya Math. J. 81 (1981), pp. 153-175. MR607081 (82f:14036)

[Mu2] S. MukAI, Fourier functor and its application to the moduli of bundles on an abelian variety, in Algebraic geometry, Sendai, 1985, Adv. Stud. Pure Math., vol. 10, NorthHolland, Amsterdam, 1987, pp. 515-550. MR946249 (89k:14026)

[Na] J.C. Naranjo, Fourier transform and Prym varieties, J. Reine Angew. Math., 560 (2003), pp. 221-230. MR.1992806 (2004e:14050)

[PP] G. PARESChI, M. PopA, Generic vanishing and minimal cohomology classes on abelian varieties, Math. Ann. 340 (2008), pp. 209-222. MR2349774(2008i:14070)

[Ra] Z. RAn, On a Theorem of Martens, Rend. Sem. Mat. Univ. Politec. Torino, 44 (1986), pp. 287-291. MR906019 (88k:14017)

[SV] R. SMith, R. VARLEY, A Torelli theorem for special divisor varieties $X$ associated to doubly covered curves $\widetilde{C} / C$, Internat. J. Math., 13 (2002), pp. 67-91. MR.1884563 (2003b:14037)

[W1] G. Welters, A theorem of Gieseker-Petri type for Prym varieties, Ann. Sci. École Norm. Sup. 18 (1985), pp. 671-683. MR839690 (88a:14034)

[W2] G. Welters, The surface $C-C$ and the second order theta functions, Acta Math., 157 (1986), pp. 1-22. MR857677 (87j:14048)

[W3] G. Welters, Curves of twice the minimal class on principally polarized abelian varieties, Nederl. Akad. Wetensch. Indag. Math., 49 (1987), pp. 87-109. MR883371 (88c:14061)

[W4] G. Welters, Recovering the Curve Data from a General Prym Variety, Amer. J. of Math., 109 (1987), pp. 165-182. MR878204 (88c:14041)

Departament d’Àlgebra i Geometria, Facultat de Matemàtiques, Universitat de BarCelona, Gran Via 585, 08007 Barcelona, Spain

E-mail address: marti.lahoz@ub.edu

Current address: Département de Mathématiques d'Orsay, Université Paris Sud 11, Bâtiment 425, F-91405 Orsay, France

Departament d'Àlgebra i Geometria, Facultat de Matemàtiques, Universitat de BarCelona, Gran Via 585, 08007 Barcelona, Spain

E-mail address: jcnaranjo@ub.edu 